\title{
THE BIFURCATION THEOREM ON THE PROBLEM OF THERMAL CONVECTION AND CONTAMINANT TRANSPORT IN UNDERGROUND WATER
}

\author{
Ngo HuY CaN*, TRAN THU HA** \\ * Institute of Mechanics, NCST of Vietnam \\ ** Institute of Applied Mechanics, NCST of Vietnam
} ABSTRACT. In the paper the bifurcation theorem on the problem of thermal convection
and contaminant transport in underground water is proven.

1. Formulation of the problem

Equations of the problem on thermal convection and contaminant transport in underground water assume the dimensionless form [1]

$$
\begin{aligned}
& \bar{v}=-\alpha \nabla p+R_{T} T \bar{\gamma}-R_{C} C \bar{\gamma} \\
& \frac{\partial T}{\partial t}+\bar{v} \cdot \nabla T=\Delta T, \\
& \varepsilon \frac{\partial C}{\partial t}+\bar{v} \cdot \nabla C=\frac{1}{L e} \Delta C, \\
& \operatorname{div} \bar{v}=0, \text { in } \Omega,
\end{aligned}
$$

with the boundary conditions:

$$
v_{n}=0, \quad T=T_{1}, \quad C=C_{1} \quad \text { on } \partial \Omega
$$

and initial conditions

$$
T_{0}=T\left(x_{0}, y_{0}, z_{0}\right), \quad C_{0}=C\left(x_{0}, y_{0}, z_{0}\right) \quad \text { at } t=t_{0}
$$

where the following notations are used: $\bar{v}$ denotes the velocity, $p$ - pressure, $T$ temperature, $C$ - concentration $\alpha=\frac{k}{H^{2} \nu}, k$ - coefficient of permeability, $H$ - a length scale, $\nu$ - coefficient of kinematical viscosity, $\varepsilon=\frac{\phi}{\sigma}, \phi$ - porosity, $\sigma$ - heat capacity of porous media, $\bar{\gamma}$ - unit vector of the vertical upward axis $O x_{3}$ in the 
Cartesian coordinate system $O x_{1} x_{2} x_{3}, L e$ - Lewis number, $R_{T}$ - thermal Rayleigh number, $R_{C}$ - concentration Rayleigh number.

In [2] it is proved that there exists a mechanical equilibrium in the fluid:

$$
\begin{aligned}
\bar{v} & =0, \\
C_{0} & =-A_{0}^{C} x_{3}+B_{0}^{C}, \\
T_{0} & =-A_{0}^{T} x_{3}+B_{0}^{T},
\end{aligned}
$$

$A_{0}^{C}, B_{0}^{C}, A_{0}^{T}, B_{0}^{T}$ the constants.

In [2] the existence theorem and the spectrum theorem of the linear problem are proved.

In this paper we prove the bifurcation theorem of the problem

$$
\begin{aligned}
& \bar{v}=-\alpha \nabla p-R_{C} C \bar{\gamma}+R_{T} T \bar{\gamma}, \\
& \bar{v} \cdot \nabla T-\bar{v} \cdot \bar{\gamma}=\Delta T, \\
& \bar{v} \cdot \nabla C-\bar{v} \cdot \bar{\gamma}=\frac{1}{L e} \Delta C, \\
& v_{n}=0, \quad T=0, \quad C=0 \text { on } \Omega .
\end{aligned}
$$

2. The bifurcation theorem

From (1.1) - (1.4), (1.7) we can obtain

$$
\begin{aligned}
& \bar{v}=-\alpha \nabla p \div R_{C} C \bar{\gamma}+R_{T} T \bar{\gamma}, \\
& \frac{\partial T}{\partial t}-\bar{v} \cdot \bar{\gamma}=\Delta T \\
& \dot{\varepsilon} \frac{\partial C}{\partial t}-\bar{v} \cdot \bar{\gamma}=\frac{1}{L e} \Delta C, \\
& \operatorname{div} \bar{v}=0 .
\end{aligned}
$$

Suppose that

$$
\begin{aligned}
& \bar{v}\left(x_{1}, x_{2}, x_{3}, t\right)=\bar{v}\left(x_{1}, x_{2}, x_{3}\right) e^{-\lambda t}, \\
& p\left(x_{1}, x_{2}, x_{3}, t\right)=p\left(x_{1}, x_{2}, x_{3}\right) e^{-\lambda t}, \\
& C\left(x_{1}, x_{2}, x_{3}, t\right)=C\left(x_{1}, x_{2}, x_{3}\right) e^{-\lambda t} \\
& T\left(x_{1}, x_{2}, x_{3}, t\right)=T\left(x_{1}, x_{2}, x_{3}\right) e^{-\lambda t}
\end{aligned}
$$


From (2.1)-(2.4) we obtain

$$
\begin{aligned}
& \bar{v}=-\alpha \nabla p-R_{C} C \bar{\gamma}+R_{T} T \bar{\gamma}, \\
& -\lambda T-\bar{v} \cdot \bar{\gamma}=\Delta T, \\
& -\lambda \varepsilon C-\bar{v} \cdot \bar{\gamma}=\frac{1}{L e} \Delta C, \\
& \operatorname{div} \bar{v}=0, \\
& v_{n}=0, \quad C=0, \quad T=0 \quad \text { on } \partial \Omega .
\end{aligned}
$$

The problem (2.5)-(2.9) is equivalent to the following operator equations in the spaces $\tilde{L}_{2}(\Omega)$ and $H_{2}(\Omega)[3]$

$$
\begin{aligned}
& \bar{v}=-R_{C} B_{12} C+R_{T} B_{12} T, \\
& \lambda T+B_{21} \bar{v}=A T, \\
& \lambda \varepsilon C+B_{21} \bar{v}=\frac{1}{L e} A C,
\end{aligned}
$$

where $L_{2}(\Omega)$ is the space of quadratically integrable vector functions in $\Omega, H_{2}(\Omega)$ is the space of quadratically integrable scalar functions in $\Omega$

$$
\begin{aligned}
& \tilde{L}_{2}(\Omega)=\left\{\bar{v}, \bar{v} \in L_{2}(\Omega), \operatorname{div} \bar{v}=0 \text { in } \Omega v_{n}=0 \text { on } \partial \Omega\right\}, \\
& B_{12} C \equiv \Pi C \bar{\gamma}, \quad B_{12} T \equiv \Pi T \bar{\gamma}, \quad B_{21} \bar{v} \equiv(\bar{v} \cdot \bar{\gamma}),
\end{aligned}
$$

$\Pi$ denotes an operator of orthogonal projection to $\tilde{L}_{2}(\Omega)$. The operator $A$ is selfadjoint positive definite in $H_{2}(\Omega)$, its inverse operator is positive and compact $[3]$.

In [2] it is proved that if $R_{T}>0, R_{C}<0$ or $R_{T}<0, R_{C}>0$ all normal disturbances vary monotonically with time-being either damped or amplified (monotonicity principle for disturbances) (see [4]).

Putting $\lambda=0$ in $(2.10)-(2.12)$ we obtain

$$
\begin{aligned}
& \bar{v}=-R_{C} B_{12} C+R_{T} B_{12} T, \\
& B_{21} \bar{v}=A T, \\
& B_{21} \bar{v}=\frac{1}{L e} A C .
\end{aligned}
$$

From (2.13)-(2.15) we have

$$
\bar{v}=-R_{C} L e B_{12} A^{-1} B_{21} \bar{v}+R_{T} B_{12} A^{-1} B_{21} \bar{v}
$$


or

$$
\begin{aligned}
& \bar{v}=\left(R_{T}-L e R_{c}\right) A \bar{v} \\
& A=B_{12} A^{-1} B_{21}
\end{aligned}
$$

Since the operator $A^{-1}$ is positive and compact, operators $B_{12}, B_{21}$ are adjoint and bounded, so the operator $A$ is self-adjoint positive and compact and the eigenvalues $R=R_{T}-L e R_{C}$ are discrete and real.

The eigenvalues $R=R_{T}-L e R_{C}$ of the problem (2.16) are called the critical values of the problem (2.10)-(2.12). We have

Lemma. The critical values $R=R_{T}-L e R_{C}$ of the problem (2.10)-(2.12) are discrete and real.

We rewrite the problem (1.8)-(1.11) in the equivalent operator equations:

$$
\begin{aligned}
& \bar{v}=-R_{C} B_{12} C+R_{T} B_{12} T, \\
& (\bar{v} \cdot \nabla T)-B_{21} \bar{v}=-A T, \\
& (\bar{v} \cdot \nabla C)-B_{21} \bar{v}=-\frac{1}{L e} A C
\end{aligned}
$$

or

$$
\begin{aligned}
\bar{v} & =-R_{C} B_{12} C+R_{T} B_{12} T \\
T & =-A^{-1}(\bar{v} \cdot \nabla T)+A^{-1} B_{21} \bar{v} \\
C & =-\operatorname{Le} A^{-1}(\bar{v} \cdot \nabla C)+\operatorname{Le} A^{-1} B_{21} \bar{v}
\end{aligned}
$$

From (2.18), (2.19) it follows

$$
\begin{aligned}
& \left(E+A_{v 1}\right) T=A^{-1} B_{21} \bar{v} \\
& \left(E+A_{v 2}\right) C=L e A^{-1} B_{21} \bar{v}
\end{aligned}
$$

where

$$
\begin{aligned}
& A_{v 1} T \equiv A^{-1}(\bar{v} \cdot \nabla T) \\
& A_{v 2} C \equiv \operatorname{LeA}^{-1}(\bar{v} \cdot \nabla C)
\end{aligned}
$$

As in [5] it is easy to prove that the operators $\left(E+A_{v 1}\right)$ and $\left(E+A_{v 2}\right)$ get an inverse operator and $A^{-1}$ is its differential Fresher at $\bar{v}=0$ :

$$
\begin{aligned}
& \left(E+A_{v 1}\right)^{-1} A^{-1}=A^{-1}+G_{1}(\bar{v}), \\
& \left(E+A_{v 2}\right)^{-1} A^{-1}=A^{-1}+G_{2}(\bar{v})
\end{aligned}
$$


where

$$
\begin{gathered}
\lim _{\|\bar{v}\| \rightarrow 0} \frac{\left\|G_{1}(\bar{v})\right\|_{H_{2,0}^{1}}(\Omega)}{\|\bar{v}\| \tilde{W}_{2,0}^{1}(\Omega)}=0, \\
\lim _{\|\bar{v}\| \rightarrow 0} \frac{\left\|G_{2}(\bar{v})\right\|_{H_{2,0}^{1}}(\Omega)}{\|\bar{v}\| \tilde{W}_{2,0}^{1}(\Omega)}=0, \\
H_{2,0}^{1}(\Omega)=\left\{q \in H_{2}(\Omega), \nabla q \in H_{2}(\Omega), q=0 \text { on } \partial \Omega\right\}, \\
W_{2,0}^{1}(\Omega)=H_{2,0}^{1}(\Omega) \times H_{2,0}^{1}(\Omega) \times H_{2,0}^{1}(\Omega), \\
\widetilde{W}_{2,0}^{1}(\Omega)=\left\{\bar{v} \in W_{2,0}^{1}(\Omega), \operatorname{div} \bar{v}=0, \bar{v}=0 \text { on } \partial \Omega\right\} .
\end{gathered}
$$

From (2.20)-(2.23) it follows

$$
\begin{aligned}
& T=\left(A^{-1}+G_{1}(\bar{v})\right) B_{21} \bar{v} \\
& C=\operatorname{Le}\left(A^{-1}+G_{2}(\bar{v})\right) B_{21} \bar{v}
\end{aligned}
$$

Putting (2.26), (2.27) into (2.17) we get

$$
\begin{aligned}
\bar{v} & =-L e R_{C} B_{12}\left(A^{-1}+G_{2}(\bar{v})\right) B_{21} \bar{v}+R_{T} B_{12}\left(A^{-1}+G_{1}(\bar{v})\right) B_{21} \bar{v} \\
& =\left(R_{T}-L e R_{C}\right) B_{12} A^{-1} B_{21} \bar{v}-L e R_{C} B_{12} G_{2}(\bar{v}) B_{21} \bar{v}+R_{T} B_{12} G_{1}(\bar{v}) B_{21} \bar{v}
\end{aligned}
$$

or

$$
\begin{aligned}
\bar{v} & =R A+Q(\bar{v}) \\
Q(\bar{v}) & \equiv-L e R_{C} B_{12} G_{2}(\bar{v}) B_{21} \bar{v}+R_{T} B_{12} G_{1}(\bar{v}) B_{21} \bar{v}
\end{aligned}
$$

$B_{12}, B_{21}$ are the bounded operators; using $(2.24),(2.25)$ we get

$$
\lim \frac{\|Q(\bar{v})\|_{H_{2,0}^{1}(\Omega)}}{\|\bar{v}\|_{\tilde{W}_{2,0}^{1}(\Omega)}}=0
$$

This implies that the operator $A$ is a differential Fresher of the operator $R A+Q$ at $\bar{v}=0$.

Using the theorem of Krasnoselskii [6] we get

Theorem. Let $R_{i}=R_{T_{i}}-$ Le $R_{C_{i}}$ which are eigenvalues of problem (2.13)-(2.15). If $R_{T}$ and $R_{C}$ are such that $R=R_{T}-$ Le $R_{C}<R_{1}$, then the problem (1.8)-(1.11) gets only a trivial solution. If $R_{1}=R_{T_{1}}-$ Le $R_{C_{1}}$ gets odd multiplicity then $R_{1}$ is a bifurcation point of the problem (1.8)-(1.11). 
This publication is completed with financial support from the Council for Natural Sciences of Vietnam.

\section{REFERENCES}

1. Harr M. E. Ground water and see page, Mc Graw-Hill, 1962.

2. Ngo Huy Can, Ta Ngoc Cau, Nguyen The Hung, Tran Thu Ha. Convection and contaminant transport in ground water. Proceedings of National Conference of Mechariics, 1997.

3. Kopachevski N. D., Krein S. G., Ngo Huy Can. Operator method in linear hydrodynamics, Nauka, Moscow, 1989.

4. Gershuni G. S., Zhukhoviskii E. M. Convective stability of incompressible fluid, Nauka, Moscow, 1972.

5. Iudovich V. I., Ukhovskii M. P. On equations of stationary convection. J. PMM 27, No 2 (1963), 295-300.

6. Krasnosebskii M. A. Topology methods in theory of nonlinear integral equations, Nauka, Moscow, 1956.

Received May 19, 2000

ĐỊNH LÝ PHÂN NHÁNH NGHIỆM CỬA BÀI TOÁN VỀ LAN TRUYỀN NHIỆT, CHẤT TRONG CÁC VİA NƯỚC NGẦM

Bài báo đã chứng minh định lý phân nhạ́nh nghiệm của bài toán về lan truyền nhiệt chất trong các vỉa nước ngầm.

* Institute of Mechanics, NCST of Vietnam

264 Doi Can, Ba Dinh, Hanoi.

** Institute of Applied Mechanics, NCST of Vietnam

Dien Bien Phu, Ho Chi Minh City 